\title{
Protective effect of edaravone for tourniquet-induced ischemia-reperfusion injury on skeletal muscle in murine hindlimb
}

Kazuichiro Hori ${ }^{1}$, Masaya Tsujii ${ }^{*}$, Takahiro lino ${ }^{1}$, Haruhiko Satonaka', Takeshi Uemura', Koji Akeda', Masahiro Hasegawa', Atsumasa Uchida ${ }^{2}$ and Akihiro Sudo ${ }^{1}$

\begin{abstract}
Background: Studies have shown that ischemia-reperfusion (I/R) produces free radicals leading to lipid peroxidation and damage to skeletal muscle. The purposes of this study were 1) to assess the histological findings of gastrocnemius muscle (GC) and tibialis anterior muscle (TA) in I/R injury model mice, 2) to histologically analyze whether a single pretreatment of edaravone inhibits I/R injury to skeletal muscle in murine models and 3) to evaluate the effect of oxidative stress on these muscles.
\end{abstract}

Methods: C57BL6 mice were divided in two groups, with one group receiving $3 \mathrm{mg} / \mathrm{kg}$ intraperitoneal injections of edaravone (I/R + Ed group) and the other group receiving an identical amount of saline (I/R group) 30 minutes before ischemia. Edaravone (3-methy-1-pheny1-2-pyrazolin-5-one) is a potent and novel synthetic scavenger of free radicals. This drug inhibits both nonenzymatic lipid peroxidation and the lipoxygenase pathway, in addition to having potent antioxidant effects against ischemia reperfusion. The duration of the ischemia was 1.5 hours, with reperfusion at either 24 or 72 hours ( 3 days). Specimens of gastrocnemius (GC) and anterior tibialis (TA) were removed for histological evaluation and biochemical analysis.

Results: This model of I/R injury was highly reproducible in histologic muscle damage. In the histologic damage score, the mean muscle fibers and inflammatory cell infiltration in the I/R + Ed group were significantly less than the corresponding values of observed in the I/R group. Thus, pretreatment with edaravone was observed to have a protective effect on muscle damage after a period of $\mathrm{I} / \mathrm{R}$ in mice. In addition, the mean muscle injury score in the I/R + Ed group was also significantly less than the I/R group. In the I/R + Ed group, the mean malondialdehyde (MDA) level was lower than in the I/R group and western-blotting revealed that edaravone pretreatment decreased the level of inducible nitric oxide synthase (iNOS) expression.

Conclusions: Edaravone was found to have a protective effect against I/R injury by directly inhibiting lipid peroxidation of the myocyte by free radicals in skeletal muscles and may also reduce the secondary edema and inflammatory infiltration incidence of oxidative stress on tissue.

Keywords: Ischemia-reperfusion injury, Skeletal muscle, Free radical scavenger, Edaravone, iNOS

\footnotetext{
* Correspondence: m-t727@clin.medic.mie-u.ac.jp

'Department of Orthopaedic Surgery, Graduate School of Medicine Mie

University, 2-174 Edobashi, Tsu city Mie prefecture 514-8507, Japan

Full list of author information is available at the end of the article
} 


\section{Background}

Ischemia-reperfusion ( $\mathrm{I} / \mathrm{R})$ injury refers to tissue damage caused when blood returns to a tissue after a period of ischemia [1]. Reperfusion triggers a cascade of acute inflammatory events, leading to cellular death and resulting in tissue dysfunction and necrosis [1]. Most research concerning I/R injury has focused on the heart, kidney, liver, brain, lung and intestines since these tissues are considered vital organs [2-4]. I/R injury to skeletal muscles is also a serious problem however, and can cause local disturbances as well as systemic problems such as crush syndrome due to leakage from muscle cells into circulating blood. I/R injuries of skeletal muscles are commonly seen in a variety of trauma and other injuries including muscle damage, transplantations, plastic surgery and limb surgery with extended tourniquet application [5-7].

Although the pathophysiology of $I / R$ injuries has not been fully elucidated due to complex interactions of inflammatory and immunologic signaling pathways [8], it is clear that accumulated free radicals and anaerobic metabolites during ischemia cause the migration of white blood cells and the release of inflammatory factors such as interleukin and free radicals in the reperfusion phase [9-13]. These compounds eventually damage muscle tissues, resulting in irrecoverable muscle damage and general complications [14-16]. One of the strategies of treatment for $I / R$ injury likely to be considered is scavenging free radicals, because $I / R$ injury can activate pathways generating reactive oxygen species with the ability to break down cell membranes [14,17]. In fact, there have been several reports in which a scavenger of free radicals was effective in reducing I/R injuries. Elmali et al. stated that resveratrol, which was previously shown to have free radical scavenging and antioxidant properties in various tissues, protected the skeletal muscles against I/R injuries [18]. Ozyurt et al. described how caffeic acid phenethyl ester (CAPE) protected skeletal muscles from reperfusion injury, and how this protective effect can probably be ascribed to CAPE's free radical scavenging activity [19].

Edaravone (3-methy-1-pheny1-2-pyrazolin-5-one) is a potent and novel synthetic scavenger of free radicals. This drug inhibits both nonenzymatic lipid peroxidation and the lipoxygenase pathway, in addition to having potent antioxidant effects against ischemia reperfusion-induced vascular endothelial cell injury, delayed neuronal death, brain edema, and concomitant neurological deficits [20-23]. In Japan, edaravone was approved in 2002 for use in treating acute brain infarctions and has been reported to have antistroke neuro-protective effects [20-23]. Therefore, we hypothesized that edaravone could protect skeletal muscles from I/R injury in an in vivo model.

The purposes of this study were 1) to assess the histological findings of gastrocnemius muscle (GC) with many slow fibers and tibialis anterior muscles (TA) with many fast fibers in using the Crawford model of $I / R$ injury [5], 2) to histologically analyze whether a single pretreatment of edaravone inhibits $\mathrm{I} / \mathrm{R}$ injury on skeletal muscle in these mouse models, and 3) to evaluate the effect of oxidative stress on these muscles.

\section{Methods}

\section{Subjects}

Forty C57BL6 mice (8-10 w, male, 22-28 g; SLC, Hamamatsu, Japan) were used in all experiments of this study. The animals were housed in a temperature-controlled environment and maintained on a 12 hour light-dark cycle with food and water available ad libitum. The experimental protocol was approved by the committee of animal research at Mie University.

\section{Animal models}

Animals were deeply anesthetized with an intraperitoneal injection of pentobarbital sodium $(0.05 \mathrm{mg} / \mathrm{g}$ body weight). Bands were applied to the left proximal thighs of 40 animals by $4.5 \mathrm{oz}$ orthodontic rubber bands (ORB; American Orthodontics, Sheboygan, WI) as described by Crawford et al. [5]. Intraperitoneal injection of edaravone $3 \mathrm{mg} / \mathrm{kg}$ (I/R + Ed group) or injection of the same amount of saline (I/R group) was administered 30 minutes before rubber band application. The period of the ischemia was 1.5 hours, and the rubber band was released and specimens were collected with reperfusion at either 24 hours ( $\mathrm{n}=10$ in each group) or 72 hours $(\mathrm{n}=10$ in each group). Mice remained anesthetized throughout the duration of ischemia with supplemental anesthesia (pentobarbital sodium) as needed. Then, reperfusion was performed by cutting the band with scissor. Specimens of gastrocnemius (GC), and anterior tibialis (TA) were removed. Under deep anesthesia mice were pinned and using a microscope, muscles were carefully excised. The wet weight of the resected muscles was measured.

\section{Drug administration methods}

Edaravone was provided by Mitsubishi Tanabe Pharma Corporation (Tokyo, Japan). It was dissolved in $1 \mathrm{~N} \mathrm{NaOH}$ that was titrated to $\mathrm{pH} 7.4$ with $1 \mathrm{~N} \mathrm{HCL}$ to prepare a final concentration of $0.3 \mathrm{mg} / \mathrm{ml}$. Finally, edaravone was injected intraperitoneally at $3 \mathrm{mg} / \mathrm{kg}$. Intraperitoneal injection of the same amount of saline ( $I / R$ group) was administered. The application of drugs was performed under the deep anesthesia before the ischemia by the tourniquet.

\section{Histological analysis}

The ten GC and TA samples $(\mathrm{n}=5$ in each group $24 \mathrm{~h}$, $72 \mathrm{~h}$ ) harvested from each group were immediately fixed in $4 \%$ paraformaldehyde and stored at $4^{\circ} \mathrm{C}$ overnight. 
The specimens were cut transversely at $4 \mu \mathrm{m}$ on a microtome and stained with hematoxylin and eosin (HE). After dewaxing in xylene and rehydration in graded methanol (99\% to $70 \%(\mathrm{v} / \mathrm{v}))$ followed by distilled water, $\mathrm{HE}$ staining was performed. The stained slides were examined at $200 \times$ magnification on microscopes equipped with a digital camera (BX50; Olympus, Tokyo, Japan). Using a standardized method, each muscle was divided into 15 fields for GC samples and 10 fields for TA samples that were photographed using a CoolSNAP color camera (Roper Scientific, Tuscan, AZ, USA) and analyzed with the software program RS Image (Roper Scientific).

\section{Histologic grading}

In tissue sections stained with $\mathrm{HE}$, a histological damage score was assigned on the following scales: disorganization and degeneration of the muscle fibers (0: Normal, 1: Mild, 2: Moderate, 3: Severe); Inflammatory cell infiltration (0: Normal, 1: Mild, 2: Moderate, 3: Severe) [24]. To test for reproducibility we reviewed our grading with 2 additional laboratory members unaware of the experimental design.

\section{Histologic injury severity score for muscles}

The absolute injury score of each muscle was determined by a method similar to McCormack et al. [25]. Every myocyte in all 15 (GC) or 10 (TA) photograph fields was scored. The muscle injury score was expressed as a percentage, obtained by the number of injured myocytes divided by the total number of myocytes scored within the all fields. The intraobserver and interobserver reliability correlation coefficients of muscle injuries at 2 times were excellent $(K=0.98,0.90$, respectively), as determined by the Cohen kappa correlation coefficient.

\section{Measurement of malondialdehyde (MDA)}

MDA is one of the end products of lipid peroxidation, and has been widely used to determine lipid peroxidation levels $[26,27]$. The lipid peroxidation was assessed by measuring the MDA content of tissue using a thiobarbituric acid (TBA) assay kit (Northwest Life Science Specialties LLC, Vancouver, WA, USA). The harvested muscle ( $\mathrm{n}=5$ in each group $24 \mathrm{~h}$ ) were immediately frozen in liquid nitrogen after washing with $0.9 \% \mathrm{NaCl}$. These resected muscles were then homogenized using a Cryopress (Microtech, Chiba, Japan)、 and stirred in an assay buffer (phosphate buffer, pH 7.0 with EDTA) for 1 hour. Butanol was then added to the sample to remove hemoglobin, because malondialdehyde (MDA) or MDA-like substances and TBA can react, producing a pink pigment in the TBA test reaction. The precipitate was centrifugally pelleted ( 3 minutes at
$10,000 \mathrm{~g})$, and an aliquot of the supernatant was reacted with an equal volume of TBA at $60^{\circ} \mathrm{C}$ for $60 \mathrm{~min}$. After cooling, sample absorbance at $540 \mathrm{~nm}$ was measured. The results were expressed in nmol /mg protein for tissue samples, using the standard graphic prepared according to the measurements done with a standard solution. (Solutions 0 to 4 )

\section{Western blotting}

Inducible nitric oxide synthase (iNOS) is a messenger protein involved in immune response, which produces large amounts of NO [28,29]. Expression of iNOS was analyzed by western blotting. Harvested muscle $(n=5$ in each group $24 \mathrm{~h}$ ) were frozen in liquid nitrogen, homogenized using a Cryopress (Microtech, Chiba, Japan) and stirred in the RIPA buffer (10 mMTrisHCl (PH 7.4), 1\% NP40, 0.1\% Sodium deoxycolate, 0.1\% Sodium dodecyl sulfate (SDS), 0.15M NaCl, 1mM EDTA, $10 \mathrm{ug} / \mathrm{ml}$ aprotinine) for 1 hour. The supernatants were separated by Sodium dodecyl sulfate Polyacrylamide gel electrophoresis (SDS-PAGE), transferred to nitrocellulose membranes, and immunoblotted with primary antibodies. The primary antibody was polyclonal rabbit antiserum against iNOS (Affinity BioReagents, Golden, CO, USA). The bands were visualized using the ECL Western blotting detection system (GE Healthcare UK Ltd., Buckinghamshire, England) and were detected by an LAS-4000 imager (Fujifilm, Tokyo, Japan).

\section{Statistical analysis}

Stat View 5.0 for Windows (SAS Institute, NC, USA) was used for statistical analysis. Data were analyzed using the Mann-Whitney $U$-test or by calculating Spearman's coefficient of rank correlation. Values of $\mathrm{p}<0.05$ were deemed statistically significant.

\section{Results}

The degree of muscle injuries in Crawford models was highly reproducible with few histological differences between subjects

None of the animals died and none required respiratory support or life support medication during experimental procedures in the $1.5 \mathrm{~h}$ model for this study. Histologically, uninjured muscle fibers were characterized as having well defined borders, consistent texture, and uniformity throughout the muscle fiber without holes or breaks. On the other hand, both GC and TA muscles in the I/R group exhibited destruction of muscle fibers with edema and inflammatory cell infiltration 24 hours after reperfusion. In addition, these findings were more pronounced 3 days after reperfusion (Figure 1A,B). Disorganization of muscle fibers and inflammatory cell infiltration was more severe in the center of muscles compared to in the periphery. 


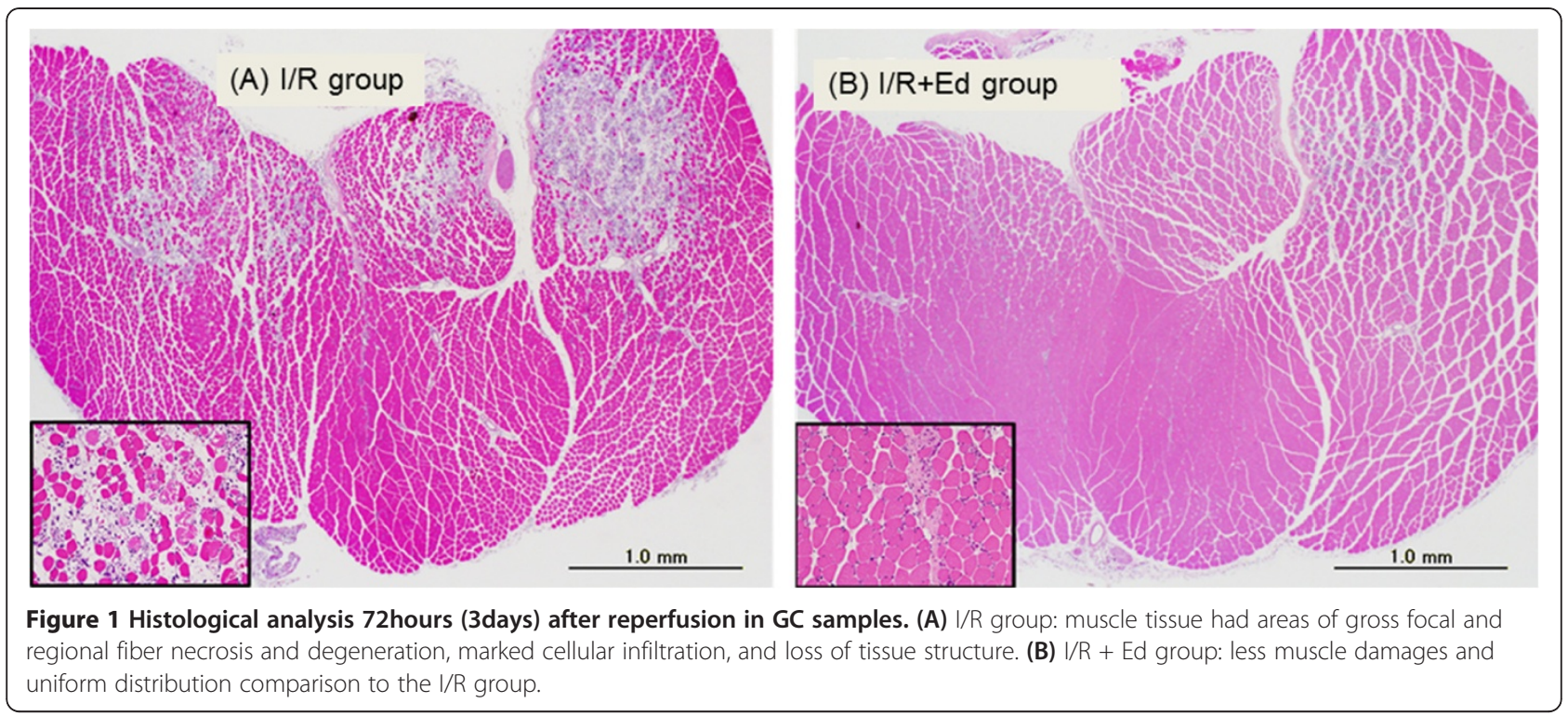

There was no thrombosis in arteries and also no Deep Venous Thrombosis (DVT) observed. The degree of injuries was largely uniform in most subjects, and all subjects had a high histologic damage score 3 days after I/R injury. This model of $I / R$ injury was highly reproducible in histologic muscle damage just as in the study by Crawford et al. [5].

\section{Application of edaravone significantly inhibited I/R injury of skeletal muscles in histological analysis}

In contrast to the muscle tissues observed after I/R injury in the $I / R$ group (Figure 1A), damage to muscle fibers was rarely observed in the I/R + Ed group. There were however some cases of inflammatory cell infiltration and edema between muscle fibers (Figure 1B). In the histologic damage score, the muscle fibers and inflammatory cell infiltration in the I/R + Ed group were $2.31 \pm 0.569$ for GC and $2.22 \pm 0.465$ for TA, both of which were significantly less than the corresponding values of $5.55 \pm 0.501$ (GC) and $4.92 \pm 0.853$ (TA) observed in the $\mathrm{I} / \mathrm{R}$ group. $(\mathrm{P}<0.001)$ (Figure 2A) Thus, pretreatment with edaravone was observed to have a protective effect on muscle damage after a period of $\mathrm{I} / \mathrm{R}$ in mice. In addition, the mean muscle injury score in the I/R + Ed group was $11.1 \%(116 / 1051)$ in GC and 9.1\% (83/903) in TA, also significantly less than the $33.4 \%(363 / 1085)$ and $20.9 \%(193 / 923)$ seen in the I/R group. $(\mathrm{P}<0.001)$ The difference was also present 3 days after reperfusion, in which application of edaravone inhibited muscle damage from the $33.7 \%(394 / 1179)$ in GC and $19.7 \%(192 / 947)$ in TA seen in the I/R group to 7.9\% (93/1178) and 6.5\% (62/957). $(\mathrm{P}<0.001)$ (Figure 2B) Histological findings indicate that the scavenging of free radicals by edaravone may inhibit inflammatory cascades. (Figure 3A,B)
The scavenging of free radicals by edaravone not only directly inhibited I/R injury to skeletal muscles but also protected myocytes via indirect transduction of oxidative stress

The extent of lipid peroxidation was determined by MDA analysis in the I/R affected muscles. The mean MDA level in the muscle tissues of the $\mathrm{I} / \mathrm{R}$ group was $1.8 \mathrm{nmol} / \mathrm{mg}$ protein in GC and $2.6 \mathrm{nmol} / \mathrm{mg}$ protein in TA whereas it was $0.9 \mathrm{nmol} / \mathrm{mg}$ protein in $\mathrm{GC}$ and $0.7 \mathrm{nmol} / \mathrm{mg}$ protein in TA of the I/R + Ed group. (Figure 4) There was a tendency towards lower peroxidation levels observed in the GC samples of the I/R + Ed group, but it was not statistically significant. $(\mathrm{p}=0.08)$ The difference in the TA peroxidation levels however was significantly lower in the I/R + Ed group. $(\mathrm{p}<0.05)$ In addition, western-blotting revealed that pretreatment of edaravone decreased the level of iNOS expression. (Figure 5) This result suggests that scavenging of free radicals could inhibit the injuries caused by oxidative stress to myocytes [30].

\section{Discussion}

$I / R$ injuries are defined as the paradoxical exacerbation of cellular dysfunction and death following the restoration of blood flow to previously ischemic tissues [1]. Re-establishment of blood flow is essential to salvage ischemic tissues; however reperfusion itself causes further damage to the ischemic tissue, threatening function and viability of the organ. $\mathrm{I} / \mathrm{R}$ injuries occur in a wide range of organs including the heart, kidney, liver, brain and intestines heart, liver, brain, kidney, colon and skeletal muscles [2-4,31]. Examples of $I / R$ injuries of skeletal muscles in this study can arise as a consequence of a range of vascular events including thrombolytic therapy, organ transplantation, limb trauma, or aortic clamping 


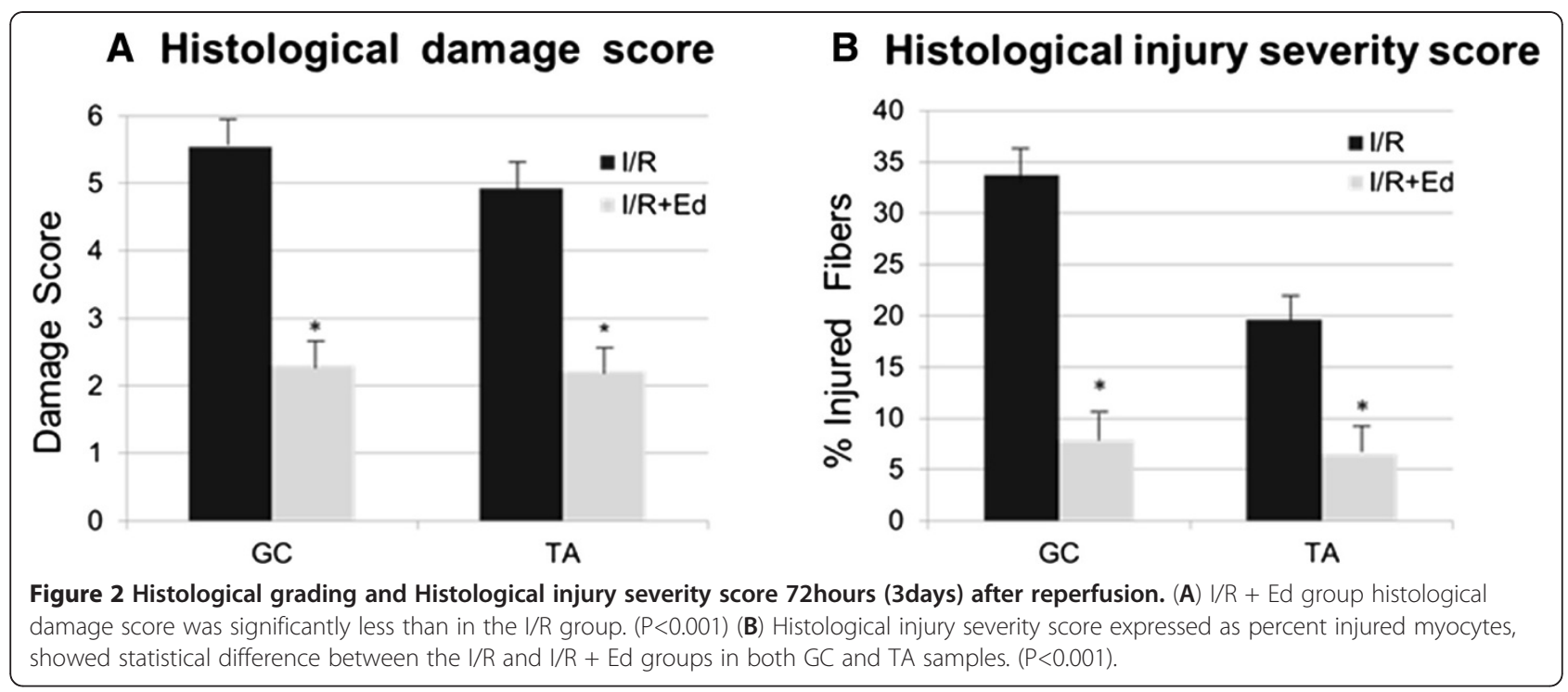

during repair of abdominal aortic aneurysms. The resulting tissue destruction and edema can lead to further worsening of the physiology and increased microvessel constriction leading to compartment syndrome [32-34]. In addition, if a large volume of tissue falls into ischemia, subsequent revascularization and hence reperfusion of the ischemic tissue will also induce systemic effects on distant organs, leading to a multi-system organ failure called crush syndrome [35,36]. Effective therapies counteracting these complications are not yet available in clinical settings, though there have been a number of reports regarding treatment for I/R injuries of skeletal muscles [37-39].

Despite such studies devoted to examining I/R injury of skeletal muscle and its underlying mechanisms, no universally accepted model for inducing skeletal muscle $\mathrm{I} / \mathrm{R}$ injury has evolved. The Crawford model was used for the tourniquet-induced hindlimb $I / R$ injury in this study [5]. Another model put forth by McGivney, using a hemorrhoidal ligator (MHL) band has been widely used as tourniquet-induced hindlimb $\mathrm{I} / \mathrm{R}$ injury model in mice [37]. The MHL model, however, has been criticized for its inability to control for nonspecific neuromuscular damage due to the crushing force of the rubber band on the underlying tissue. Additionally, the tension gradually decreases in this band. On the other hand, the model using an orthodontic rubber band (ORB) reported by Crawford appears to be superior to the MHL band model because ORBs produce complete, consistent, and reproducible ischemia in murine models of $I / R$ using significantly less tension than the MHL band. Our histological analysis also showed that there was little difference in the degree of injury of muscle fibers, indicating that this simple model was likely to be suitable for evaluation of the effects of treatment for skeletal muscles of I/R injury.

While there have been several clinical strategies designed to reduce the effects of $I / R$ injuries, none of them have been broadly successful in attenuating detrimental

\section{A GC samples}

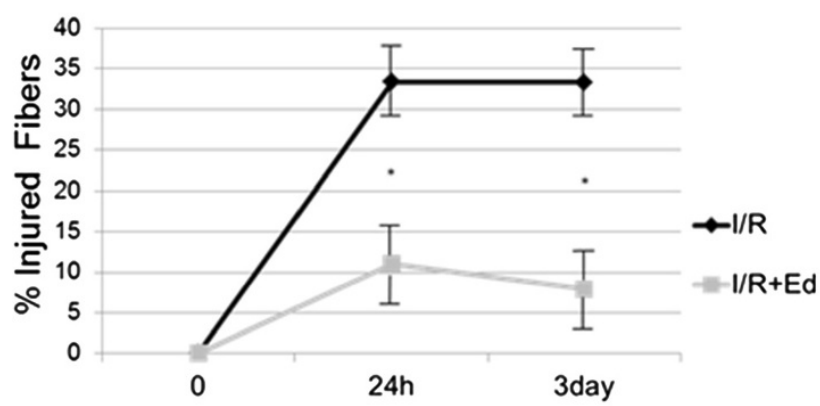

\section{B TA samples}

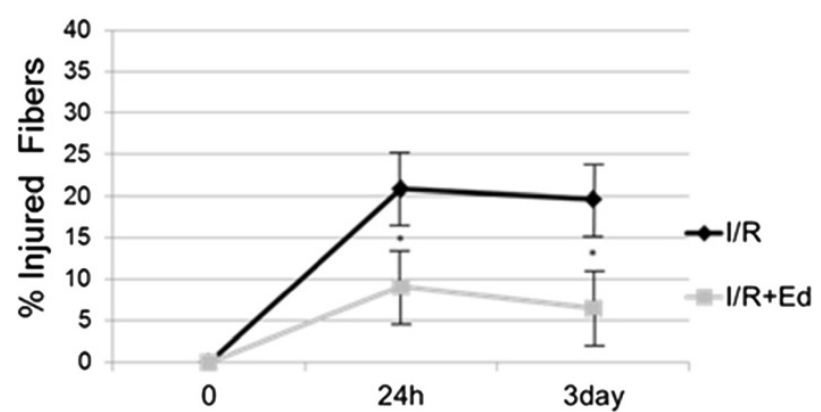

Figure 3 Histological assessment percentage injury over time. (A) GC samples: The I/R + Ed group showed a significantly a lower injury percentage at $24 \mathrm{~h}$, and continuously decreased injury rate at $72 \mathrm{~h}$. ( $\mathrm{P}<0.001)$ (B) TA samples: The I/R + Ed group similary showed significantly a lower injury percentage and a decreasing injury rate at the $72 \mathrm{~h}$ sample time. $(\mathrm{P}<0.001)$. 


\section{Measurement of malondialdehyde (MDA)}

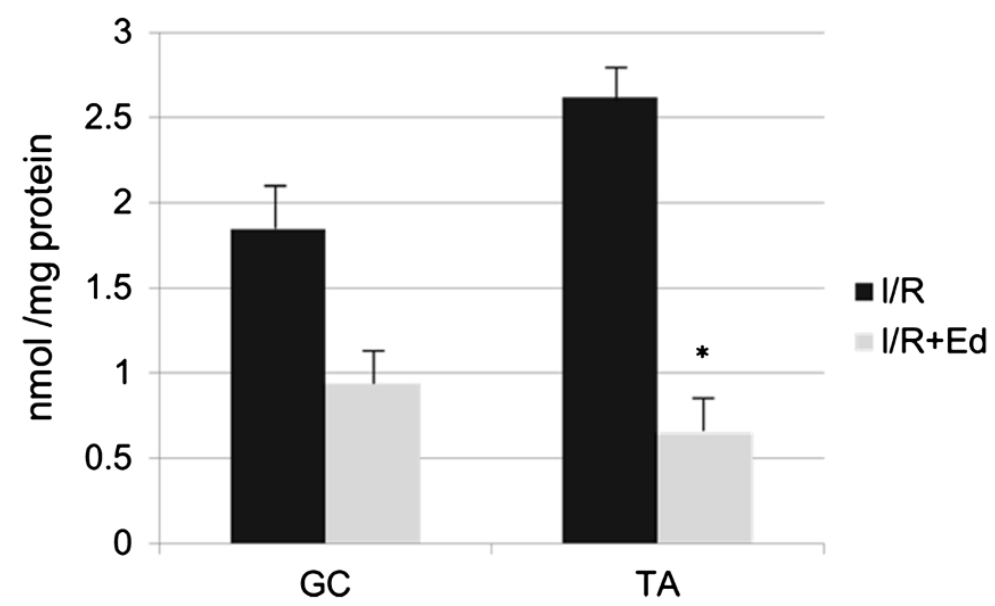

Figure 4 Malondialdehyde (MDA) concentration in GC and TA 24hours (1day) after reperfusion. The I/R + Ed group, the mean MDA level was lower than in the I/R group. There was a tendency towards lower peroxidation levels observed in the GC samples of the I/R + Ed group, but it was not statistically significant. $(p=0.08)$ The difference in the TA peroxidation levels however was significantly lower in the $1 / R+E d$ group. $(p<0.05)$

effects. These strategies include pharmacological intervention, hyperbaric oxygen, hypothermia, and pre- and postinjury conditioning [38-40]. The potential therapeutic benefits of edaravone has been previously recognized and investigated for $I / R$ injuries such as cerebral infarctions as there have been a number of reports that oxygen free radicals are involved in the pathogenesis of $I / R$ injury $[20,22,41]$. A recent Japanese multicenter randomized clinical trial has demonstrated that edaravone, administered within 72 hours after onset of ischemic stroke significantly reduces brain infarct volume and produces sustained benefits in the functional outcomes of patients compared with placebo treatment [23]. These results led to our hypothesis that edaravone, approved in Japan as a neuro-protective agent, could protect skeletal muscles from $\mathrm{I} / \mathrm{R}$ injury not only in the animal model, but also in clinical situations. Furthermore, it has been reported that application of other antioxidants has therapeutic potential for I/R injury of skeletal muscle. The present study demonstrates that pretreatment of edaravone significantly protects against $I / R$ injury of skeletal muscles in the hindlimb of mice. This study is particularly significant for showing the therapeutic benefits of a single-dose administration of edaravone 30 minutes prior to the onset of ischemia. This preischemic treatment protocol mimics the commonly seen clinical conditions of long duration tourniquet use for extremity surgery and plastic surgeries such as free muscle transfer.

\section{iNOS Expression}

TA

GC

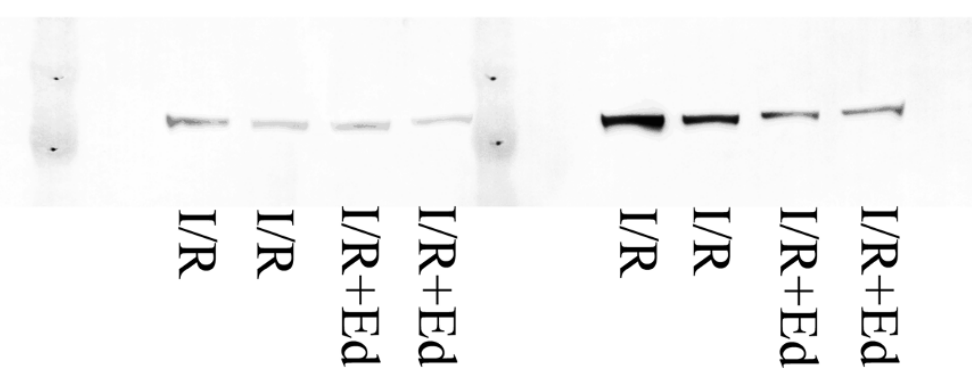

Figure 5 Immunoblotting with anti-iNOS 24hours (1day) after reperfusion. Pretreatment of edaravone decreased the level of iNOS expression. 
Infiltration of inflammatory cells was also inhibited by scavenging free radicals with edaravone. The final common pathway of $\mathrm{I} / \mathrm{R}$ injury is edema, attraction of activated leukocytes, and the formation of membrane-attack complexes, all of which lead to disruption of cell membranes resulting cell death [17]. The oxygen free radicals initiate lipid peroxidation of cell membranes, which causes cellular dysfunction and tissue necrosis. Our results showed that one dose of pretreatment edaravone efficiently reduced lipid peroxidation and neutrophil infiltration into the ischemic muscles. Therefore, the scavenging of oxygen free radicals induced immediately after ischemia-reperfusion may not only maintain tissue viability but also enable sufficient contractile function of skeletal muscle.

Furthermore, expression of iNOS, which was shown to play an important role in the pathophysiology of $\mathrm{I} / \mathrm{R}$ injury in other organs as well as inflammatory cytokine, was inhibited in the skeletal muscles treated by edaravone $[20,42]$. The skeletal muscles were also reported to be influenced by NO generated by inducible form of NOS [43]. Barker et al. demonstrated that the area of necrosis was greater in wild-type than in iNOS-knockout mice, the difference being significant after 90 minutes of ischemia [43]. NO is formed as a metabolic product the stepwise conversion of L-arginine guanidine nitrogen atoms [44]. The presence of an unpaired electron in the NO radical makes it highly reactive, with a half-life of only a few seconds and readily capable of reacting with other species such as superoxides. The reaction is catalyzed by the NOS enzyme, of which three isoforms have been identified, each having specific localizations and functions [29]. Three NOS isoforms has been characterized, each encoded by different chromosomes. Two enzyme isoforms are constitutively expressed (endothelial eNOS and neuronal nNOS), whereas one isoform is an inducible enzyme (iNOS), initially found in macrophages. iNOS levels are markedly increased in inflammatory conditions, such as autoimmune inflammatory myopathies. The inducible isoform of NOS becomes up regulated in response to inflammatory stimuli such as endotoxins, cytokines and lipid mediators. Surges in NO production mediated by iNOS are cytotoxic and have been implicated in the inflammatory destruction of tissues such as heart, kidney, liver, brain, intestine and skeletal muscle [45]. In our experiments expression of iNOS was inhibited in the skeletal muscles treated by edaravone. Therefore, directly inhibiting lipid peroxidation of the myocyte by free radicals in skeletal muscles may reduce the secondary edema and inflammatory infiltration incidence of oxidative stress on tissues.

\section{Conclusion}

The injury severity score in the $I / R+E d$ group compared to the $I / R$ group showed a significantly a lower rate both in GC and TA samples. While edaravone may have multiple effects, our data showed that pretreatment with edaravone protected muscle tissue from damage due to ischemia-reperfusion. We speculate that pretreatment of edaravone directly inhibits lipid peroxidation of myocytes by free radicals in skeletal muscles after I/R injury and indirectly protects myocytes from secondary damage due to edema, inflammatory infiltration and transduction of oxidative stress. Further studies are warranted to confirm the novel therapeutic potential edaravone may have for $I / R$ injuries of skeletal muscle.

\section{Abbreviations}

I/R: Ischemia-reperfusion; GC: Gastrocnemius; TA: Tibialis anterior; DVT: Deep venous thrombosis; Ed: Edaravone; MDA: Malondialdehyde; iNOS: Inducible nitric oxide synthase; CAPE: Caffeic acid phenethyl ester; ORB: Orthodontic rubber bands; HE: Hematoxylin and eosin; RS: Roper scientific;

TBA: Thiobarbituric acid; SDS: Sodium dodecyl sulfate; SDS-PAGE: Sodium dodecyl sulfate polyacrylamide gel electrophoresis; MHL: McGivney hemorrhoidal ligator; eNOS: Endothelial nitric oxide synthase; nNOS: Neuronal nitric oxide synthase.

\section{Competing interests}

The authors declare that they have no competing interests.

\section{Authors' contributions}

$\mathrm{KH}$ and MT were responsible for the design and coordination of the data acquisition and analysis, interpretation of the data, and the writing of the manuscript. TI and TU participated in the experimental design and techniques. HS and KA performed the pathological examinations. MH participated in statistical evaluation of the data. AU and AS did final editing. All authors read and approved the final manuscript.

\section{Acknowledgements}

We are grateful to Mitsubishi Tanabe Pharma Corporation (Tokyo, Japan), especially Akihide Nishi and Takeshi Tanaka for providing edaravone free. We thank Katsura Chiba for help the experimental techniques. This study was supported by Department of Orthopaedic Surgery, Graduate School of Medicine Mie University and the committee of animal research at Mie University.

\section{Author details}

'Department of Orthopaedic Surgery, Graduate School of Medicine Mie University, 2-174 Edobashi, Tsu city Mie prefecture 514-8507, Japan. ${ }^{2}$ Mie University, Mie, Japan.

Received: 12 December 2012 Accepted: 21 March 2013

Published: 27 March 2013

\section{References}

1. Carden DL, Granger DN: Pathophysiology of ischaemia-reperfusion injury. J Pathol 2000, 190(3):255-266.

2. Chamoun F, Burne M, O'Donnell M, Rabb H: Pathophysiologic role of selectins and their ligands in ischemia reperfusion injury. Frontiers in bioscience: a journal and virtual library 2000, 5:E103-E109.

3. de Perrot M, Liu M, Waddell TK, Keshavjee S: Ischemia-reperfusion-induced lung injury. Am J Respir Crit Care Med 2003, 167(4):490-511.

4. Tonon J, Cecchini AL, Brunnquell CR, Bernardes SS, Cecchini R, Guarnier FA: Lung injury-dependent oxidative status and chymotrypsin-like activity of skeletal muscles in hamsters with experimental emphysema. BMC Musculoskelet Disord 2013, 14:39.

5. Crawford RS, Hashmi FF, Jones JE, Albadawi H, McCormack M, Eberlin K, Entabi F, Atkins MD, Conrad MF, Austen WG Jr, et al: A novel model of acute murine hindlimb ischemia. Am J Physiol Heart Circ Physiol 2007, 292(2):H830-H837.

6. Walters TJ, Kragh JF, Kauvar DS, Baer DG: The combined influence of hemorrhage and tourniquet application on the recovery of muscle function in rats. J Orthop Trauma 2008, 22(1):47-51. 
7. Schoen M, Rotter R, Gierer P, Gradl G, Strauss U, Jonas L, Mittlmeier T, Vollmar B: Ischemic preconditioning prevents skeletal muscle tissue injury, but not nerve lesion upon tourniquet-induced ischemia. The Journal of trauma 2007, 63(4):788-797.

8. Hsieh CH, Jeng JC, Jeng SF, Wu CJ, Lu TH, Liliang PC, Rau CS, Chen YC, Lin $\mathrm{CJ}$ : MicroRNA profiling in ischemic injury of the gracilis muscle in rats. BMC Musculoskelet Disord 2010, 11:123.

9. Colletti LM, Kunkel SL, Walz A, Burdick MD, Kunkel RG, Wilke CA, Strieter RM: The role of cytokine networks in the local liver injury following hepatic ischemia/reperfusion in the rat. Hepatology 1996, 23(3):506-514.

10. Shirasugi N, Wakabayashi G, Shimazu M, Oshima A, Shito M, Kawachi S, Karahashi T, Kumamoto Y, Yoshida M, Kitajima M: Up-regulation of oxygen-derived free radicals by interleukin-1 in hepatic ischemia/reperfusion injury. Transplantation 1997, 64(10):1398-1403.

11. Yang Z, Zingarelli B, Szabo C: Crucial role of endogenous interleukin-10 production in myocardial ischemia/reperfusion injury. Circulation 2000, 101(9):1019-1026.

12. Mallick $I H$, Yang W, Winslet MC, Seifalian AM: Ischemia-reperfusion injury of the intestine and protective strategies against injury. Dig Dis Sci 2004 49(9):1359-1377.

13. McCord JM: Oxygen-derived free radicals in postischemic tissue injury. N Engl J Med 1985, 312(3):159-163.

14. Blaisdell FW: The pathophysiology of skeletal muscle ischemia and the reperfusion syndrome: a review. Cardiovasc Surg 2002, 10(6):620-630.

15. Blebea J, Cambria RA, DeFouw D, Feinberg RN, Hobson RW, 2nd Duran WN: lloprost attenuates the increased permeability in skeletal muscle after ischemia and reperfusion. J Vasc Surg 1990, 12(6):657-665. discussion 665-656.

16. Perry MO, Fantini G: Ischemia: profile of an enemy. Reperfusion injury of skeletal muscle. J Vasc Surg 1987, 6(3):231-234.

17. Eltzschig HK, Collard CD: Vascular ischaemia and reperfusion injury. British medical bulletin 2004, 70:71-86.

18. Elmali N, Esenkaya I, Karadag N, Tas F, Elmali N: Effects of resveratrol on skeletal muscle in ischemia-reperfusion injury. Ulus Travma Acil Cerrahi Derg 2007, 13(4):274-280

19. Ozyurt H, Ozyurt B, Koca K, Ozgocmen S: Caffeic acid phenethyl ester (CAPE) protects rat skeletal muscle against ischemia-reperfusion-induced oxidative stress. Vascul Pharmacol 2007, 47(2-3):108-112.

20. Yoshida H, Yanai H, Namiki Y, Fukatsu-Sasaki K, Furutani N, Tada N: Neuroprotective effects of edaravone: a novel free radical scavenger in cerebrovascular injury. CNS Drug Rev 2006, 12(1):9-20.

21. Shichinohe $H$, Kuroda $S$, Yasuda $H$, Ishikawa T, Iwai M, Horiuchi M, Iwasaki Y: Neuroprotective effects of the free radical scavenger Edaravone (MCl-186) in mice permanent focal brain ischemia. Brain research 2004, 1029(2):200-206.

22. Zhang N, Komine-Kobayashi M, Tanaka R, Liu M, Mizuno Y, Urabe T: Edaravone reduces early accumulation of oxidative products and sequential inflammatory responses after transient focal ischemia in mice brain. Stroke 2005, 36(10):2220-2225.

23. Group EABIS: Effect of a novel free radical scavenger, edaravone (MCl-186), on acute brain infarction. Randomized, placebo-controlled, double-blind study at multicenters. Cerebrovasc Dis 2003, 15(3):222-229.

24. Erkanli K, Kayalar N, Erkanli G, Ercan F, Sener G, Kirali K: Melatonin protects against ischemia/reperfusion injury in skeletal muscle. J Pineal Res 2005, 39(3):238-242.

25. McCormack MC, Kwon E, Eberlin KR, Randolph M, Friend DS, Thomas AC, Watkins MT, Austen WG Jr: Development of reproducible histologic injury severity scores: skeletal muscle reperfusion injury. Surgery 2008, 143(1): 126-133.

26. Casini AF, Ferrali M, Pompella A, Maellaro E, Comporti M: Lipid peroxidation and cellular damage in extrahepatic tissues of bromobenzene-intoxicated mice. Am J Pathol 1986, 123(3):520-531.

27. Badcock NR, Zoanetti GD, Martin ES: Nonchromatographic assay for malondialdehyde-thiobarbituric acid adduct with HPLC equivalence. Clin Chem 1997, 43(9):1655-1657.

28. Yoshida H, Kwon AH, Kaibori M, Tsuji K, Habara K, Yamada M, Kamiyama Y, Nishizawa M, Ito S, Okumura T: Edaravone prevents iNOS expression by inhibiting its promoter transactivation and mRNA stability in cytokinestimulated hepatocytes. Nitric oxide: biology and chemistry / official journal of the Nitric Oxide Society 2008, 18(2):105-112.
29. Filippin LI, Moreira AJ, Marroni NP, Xavier RM: Nitric oxide and repair of skeletal muscle injury. Nitric oxide: biology and chemistry / official journal of the Nitric Oxide Society 2009, 21(3-4):157-163.

30. Bujok G, Dyaczynska-Herman A, Jendryczko A, Mandat K, Sypniewski J: Concentration of malonic dialdehyde in the cerebrospinal fluid as a measure of the intensity of lipid peroxidation processes in intracranial hypertension in small children. Childs Nerv Syst 1996, 12(2):97-99.

31. Gute DC, Ishida T, Yarimizu K, Korthuis RJ: Inflammatory responses to ischemia and reperfusion in skeletal muscle. Mol Cell Biochem 1998, 179(1-2):169-187

32. Mubarak SJ, Pedowitz RA, Hargens AR: Compartment syndromes. Curr Orthop 1989, 3:36-40.

33. Perry MO: Compartment syndromes and reperfusion injury. Surg Clin N Am 1988, 68(4):853-864.

34. Navein J, Coupland R, Dunn R: The tourniquet controversy. The Journal of trauma 2003, 54(5 Suppl):S219-S220.

35. Odeh M: The role of reperfusion-induced injury in the pathogenesis of the crush syndrome. N Engl J Med 1991, 324(20):1417-1422.

36. Malinoski DJ, Slater MS, Mullins RJ: Crush injury and rhabdomyolysis. Critical care clinics 2004, 20(1):171-192

37. Kyriakides C, Favuzza J, Wang Y, Austen WG Jr, Moore FD Jr, Hechtman HB: Recombinant soluble P-selectin glycoprotein ligand 1 moderates local and remote injuries following experimental lower-torso ischaemia. Br J Surg 2001, 88(6):825-830.

38. Grace PA: Ischaemia-reperfusion injury. Br J Surg 1994, 81(5):637-647.

39. Wang WZ, Fang XH, Stephenson LL, Khiabani KT, Zamboni WA: Melatonin reduces ischemia/reperfusion-induced superoxide generation in arterial wall and cell death in skeletal muscle. J Pineal Res 2006, 41(3):255-260.

40. Chen CJ, Cheng FC, Liao SL, Chen WY, Lin NN, Kuo JS: Effects of naloxone on lactate, pyruvate metabolism and antioxidant enzyme activity in rat cerebral ischemia/reperfusion. Neurosci Lett 2000, 287(2):113-116.

41. Omori K, Shikata Y, Sarai K, Watanabe N, Wada J, Goda N, Kataoka N, Shikata K, Makino $\mathrm{H}$ : Edaravone mimics sphingosine-1-phosphate-induced endothelial barrier enhancement in human microvascular endothelial cells. Am J Physiol Cell Physiol 2007, 293(5):C1523-C1531.

42. Jugdutt BI: Nitric oxide and cardioprotection during ischemia-reperfusion. Hear Fail Rev 2002, 7(4):391-405.

43. Barker JE, Knight KR, Romeo R, Hurley JV, Morrison WA, Stewart AG: Targeted disruption of the nitric oxide synthase 2 gene protects against ischaemia/reperfusion injury to skeletal muscle. J Pathol 2001, 194(1):109-115.

44. Rubinstein I, Abassi Z, Coleman R, Milman F, Winaver J, Better OS: Involvement of nitric oxide system in experimental muscle crush injury. J Clin Invest 1998, 101(6):1325-1333.

45. Anggard E: Nitric oxide: mediator, murderer, and medicine. Lancet 1994 343(8907):1199-1206.

doi:10.1186/1471-2474-14-113

Cite this article as: Hori et al:: Protective effect of edaravone for tourniquet-induced ischemia-reperfusion injury on skeletal muscle in murine hindlimb. BMC Musculoskeletal Disorders 2013 14:113.

\section{Submit your next manuscript to BioMed Central and take full advantage of:}

- Convenient online submission

- Thorough peer review

- No space constraints or color figure charges

- Immediate publication on acceptance

- Inclusion in PubMed, CAS, Scopus and Google Scholar

- Research which is freely available for redistribution 